\title{
Deformation Response of Mg-Y Alloys under Dynamic Loading
}

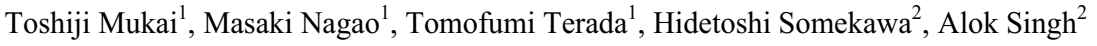 \\ ${ }^{1}$ Mechanical Engineering Department (Kobe University); 1-1 Rokkodai-cho, Nada; Kobe, Hyogo 657-8501, Japan \\ ${ }^{2}$ National Institute for Materials Science, Sengen, Tsukuba, Ibaraki 305-0047, Japan
}

Keywords: magnesium, yttrium, high strain rate, compression, deformation twin, dynamic recovery

\section{Extended Abstract}

Weight reduction of automobiles and aircrafts improves fuel economy and reduces greenhouse gas emissions. Use of $\mathrm{Mg}$ alloys may allow weight reduction because of their low densities, but adoption is hindered because they exhibit limited ductility at ambient temperatures [1]. In a previous study of fracture toughness in a $\mathrm{Mg}$ alloy, crack readily propagated near twin boundaries and resulted in poor durability [2]. It has been shown that pile-up of dislocations at the interface between the matrix and deformation twins caused stress concentration to form cracks [2]. Another study suggested that the ductility of $\mathrm{Mg}$ alloys is further limited under dynamic loading due to lowered activity of dislocations [3]. It has also been reported that a Mg-Al-Mn alloy had pronounced mechanical anisotropy at high strain rates of around $1.0 \times 10^{3} \mathrm{~s}^{-1}$ [4]. Therefore, the mechanical properties of $\mathrm{Mg}$ alloys should be evaluated accurately for applications involving possible dynamic loading.

It has been reported that addition of rare earth elements such as yttrium may improve mechanical properties over conventional $\mathrm{Mg}-\mathrm{Al}-\mathrm{Zn}$ alloys by minimizing intensity of the basal texture [59]. However, the deformation behavior of $\mathrm{Mg}$ alloys containing yttrium is not sufficiently understood at strain rates exceeding $1 \times 10^{3} \mathrm{~s}^{-1}$. This study examines a binary $\mathrm{Mg}-\mathrm{Y}$ alloy at high strain rates in compression to clarify the effect of yttrium addition on the mechanical properties. Details have been reported elsewhere [10].

This study investigated a binary Mg-0.6at.\%Y alloy. A billet was fabricated by gravity casting into a steel mold. The cast binary alloy was homogenized at $773 \mathrm{~K}$, followed by quenching in water. It was then extruded at $673 \mathrm{~K}$ at a ratio of $25: 1$. The extruded rod having a diameter of $8 \mathrm{~mm}$ was annealed at $673 \mathrm{~K}$ for $16.5 \mathrm{~h}$ to form an equiaxed grain structure with an average grain size of 42 $\mu \mathrm{m}$. As a reference material, extruded magnesium with $99.95 \%$ purity and an average grain size of $50 \mu \mathrm{m}$ was prepared. Cylindrical specimens having a height of $8 \mathrm{~mm}$ and a diameter of $4 \mathrm{~mm}$ were machined parallel to the extrusion direction. To examine the mechanical anisotropy, cylindrical specimens of height $6.5 \mathrm{~mm}$ and diameter $4 \mathrm{~mm}$ were also prepared along two directions, parallel and perpendicular to the extrusion direction. High strain rate compression tests were performed by using a split Hopkinson pressure bar (SHPB) at ambient temperature (298 K). The average strain rate was measured to be $1.4 \times 10^{3} \mathrm{~s}^{-1}$ for a specimen of $8.0 \mathrm{~mm}$ height and to be $1.7 \times 10^{3} \mathrm{~s}^{-1}$ for a specimen of
$6.5 \mathrm{~mm}$ height. The shape of the specimen during deformation was detected as a series of images by an ultra-high speed camera (Shimadzu HPV-1) with a sampling time of $4 \mu \mathrm{s}$.

By investigation of the Mg-0.6 at.\% Y alloy and comparing it with a commercial purity magnesium sample, we have found that yttrium solute contributed to enhanced compressive ductility, reduced strain hardening rate, and minimized deformation asymmetry in magnesium, even under dynamic loading. Although $\{10 \overline{1} 2\}$ twins were the predominant deformation mechanism for the Mg- $0.6 \mathrm{Y}$ alloy in the early-stage of deformation, cracks did not initiate near these twin boundaries, unlike in the conventional $\mathrm{Mg}$ alloy AZ31. This suggests occurrence of stress relaxation during deformation. Subgrain formation and c-axis rotation possibly release stress concentrations during high strain rate deformation of the Mg-Y alloy.

This work was supported in part by Toyota Motor Corp. and by a Grant-in-Aid for Scientific Research (No. 25246012) from the Ministry of Education, Culture, Sports, Science and Technology of Japan.

\section{Reference}

1. T. Mukai, H. Watanabe, K. Higashi, Mater. Sci. Technl., 2000, vol. 16, 11-12, pp. 1314-1319.

2. H. Somekawa, A. Singh and T. Mukai: Philo. Mag. Lett., 2009 vol. 89, 1, pp.2-10.

3. T. Mukai, M. Yamanoi, H. Watanabe and K. Higashi: Mater. Trans., 2001, vol. 42, 7, pp.1177-1181.

4. P. Mao, Z. Liu and C. Wang: Mater. Sci. Eng.A 2012, vol. 539, pp.13-21.

5. E.A. Ball and P.B. Prangnell, Scripta Metall. Mater., 1994, vol. 31, pp. 111-116.

6. S.R. Agnew, M.H. Yoo, C.N. Tome, Acta Mater., 2001, vol. 49, pp. 4277-4289.

7. S. Miura, S. Imagawa, T. Toyoda, K. Ohkubo and T. Mohri, Mater. Trans., 2008 vol. 49, 5, pp.952-956.

8. J. Bohlen, M. R. Nurnberg, J. W. Senn, D. Letzing, S. R. Agnew, Acta Mater., 2007, vol. 55, 6, pp. 2101-2112.

9. J. Geng, Y. B. Chun, N. Stanford, C. H. J. Davies, J. F. Nie, M. R. Barneet, Mater. Sci. Eng.A, 2011, vol. 528, 10-11, pp.36593665 .

10. M. Nagao, T. Tereda, H. Somekawa, A. Singh, T. Mukai, JOM, 2014 vol. 66, pp.305-311. 